\title{
UM ESTUDO SOBRE AS BOAS PRÁTICAS DE FABRICAÇÃO NA INDÚSTRIA DE MEDICAMENTOS VETERINÁRIOS
}

\author{
A STUDY ON GOOD MANUFACTURING PRACTICES IN THE VETERINARY \\ MEDICINAL PRODUCTS INDUSTRY
}

\author{
Leonardo Rafael Bispo - leonardorbispo@outlook.com \\ Angelita Moutin Segoria Gasparotto - angelita.gasparotto@fatectq.edu.br \\ Faculdade de Tecnologia de Taquaritinga (FATEC) - São Paulo - Brasil
}

\begin{abstract}
RESUMO
$\mathrm{Na}$ economia e no cenário econômico mundial, as empresas que processam e produzem medicamentos veterinários estão aumentando suas lucratividades e suas receitas conforme se adequam a um fator muito importante na produção de fármacos-veterinários, que é a qualidade nos seus processos e serviços. Neste ambiente, a concorrência por melhorias vem alcançando níveis de eficiência muito altos em relação às metodologias de qualidade. Uma organização que se adapta a essas necessidades cada vez mais é cobrada por órgãos vigentes e regulamentadores que, sem dúvida, não pode ficar sem essas adequações importantes. Tais órgãos têm a competência de manter estes padrões e estabelecerem as organizações padrões que sugerem a melhoria dos processos e produtos destinados a esse segmento. $\mathrm{O}$ artigo mostra as etapas de implantação em uma indústria de medicamentos veterinários, seus processos em meio à metodologia aplicada e seus desafios. Cabe salientar que os objetivos que as boas práticas de fabricação (BPF) seguem seus padrões em todo o fluxo operacional, desde o fornecimento de matérias primas até o produto acabado para os setores de expedição e logística.
\end{abstract}

Palavras-chave: Indústria de Medicamentos Veterinários, Boas Práticas de Fabricação, Qualidade.

\begin{abstract}
In the economy and in the global economic scenario, companies that process and produce veterinary drugs are increasing their profitability and revenues as they fit a very important factor in the production of veterinary drugs, which is the quality of their processes and services. In this environment, competition for improvements has been reaching very high levels of efficiency in relation to quality methodologies. An organization that adapts to these needs is increasingly being charged by governing bodies and regulators that undoubtedly can not run out of these important adaptations. These bodies have the competence to maintain these standards and establish the standard organizations that suggest the improvement of the processes and products destined to this segment. The article shows the steps of implantation in an industry of veterinary drugs, its processes amid the applied methodology and its challenges. It should be noted that the goals of Good Manufacturing Practice (GMP) follow its standards throughout the operational flow, from the supply of raw materials to the finished product for the shipping and logistics sectors.
\end{abstract}


Keywords: Veterinary Drug Industry, Good Manufacturing Practices, Quality.

\section{INTRODUÇÃO}

A procura por novos métodos de qualidade nas empresas de medicamentos veterinários fez com que o segmento crescesse a ponto de adquirir novas técnicas para suprir os setores e as necessidades nas organizações.

Os medicamentos veterinários juntamente com os medicamentos para seres humanos (saúde humana) têm suas subdivisões nas seguintes classes:

Ilustração 1: Classes de medicamentos

\begin{tabular}{l|c|c|c|l}
\hline \multicolumn{5}{c}{ Classes } \\
\hline Parasiticidas & $\begin{array}{c}\text { Biológicos } \\
\text { (Vacinas) }\end{array}$ & $\begin{array}{c}\text { Infecções } \\
\text { (Tratamentos) }\end{array}$ & $\begin{array}{c}\text { Aditivos } \\
\text { Alimentares }\end{array}$ & Outros \\
\hline
\end{tabular}

Fonte: Elaborado pelo autor (2017).

A legislação brasileira tem sua classificação de medicamentos conforme abaixo:

Referência: inovação em medicamentos (produtos novos), com o registro nos órgãos competentes e comercializados no país, tem no registro sua comprovação científica.

Similar: é o medicamento que tem a mesma concentração, farmacologia, a mesma forma farmacêutica, a via de administração e suas indicações terapêuticas iguais aos medicamentos originais. (CAPANEMA, 2007).

Salienta CAPANEMA (2007) os produtos veterinários também têm sua classificação que são agrupados por classe farmacêutica (suplementos nutricionais, fármacos e biológicos), divididos em portes de animais grandes e pequenos (pets). Contudo, existem seis classes de distribuição destes medicamentos que são bovinos, suínos equinos, aves, pequenos animais (pets) e outras classes.

Assim, o objetivo geral desta pesquisa é apresentar um estudo sobre os métodos de qualidade que é de tal importância nas estratégias competitivas das empresas. Visto que a importância do artigo é apresentar um estudo sobre as boas práticas de fabricação na indústria de medicamentos veterinários.

O método proposto no estudo foi por meio de artigo, uma proposta de melhoria nos setores de fabricação e comercialização de medicamentos veterinários e informações relevantes sobre o assunto proposto. Entretanto o conteúdo de pesquisa usado para 


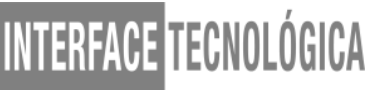

desenvolver esse trabalho foi o de artigo científico. O artigo será feito em cinco seções, que retratam o estudo proposto com um todo.

O artigo será dividido e estruturado incialmente com a introdução, com o inicio do tema e será mostrado o uso das boas práticas de fabricação (BPF) e a qualidade, o uso em processos fármacos-veterinários e as melhorias propostas da metodologia de qualidade e por fim a proposta de melhoria e as conclusões obtidas através do estudo.

\section{O ESTUdO DAS BOAS PRÁTICAS DE FABRICAÇÃO NA PRODUÇÃO DE MEDICAMENTOS VETERINÁRIOS}

O estudo é de total importância na área química, farmacêutica e de qualidade, tendo em vista que estes processos nos dias atuais não tem destaque sem o mínimo de requisitos que envolvam a qualidade nos processos, usando por completo esse método para obter efetividade.

Tem sua definição de boas práticas de fabricação levando em conta procedimentos higiênicos, sanitários e operacionais com aplicação em todo o fluxo produtivo (MAPA, 2007).

Estabelece Pinto (2012), que as empresas devem manter uma política de boas práticas de fabricação, desde projetos, instalações, pesquisa e desenvolvimento, até um sistema de controle de qualidade eficaz, inclusive o controle microbiológico para garantir produtos com alto padrão de qualidade e seguros para serem distribuídos para os consumidores.

\subsection{Controle de qualidade}

Quando se fala em qualidade nas indústrias farmacêuticas, pode se entender que é um passo indispensável nas empresas, no entanto, não se tem vantagem competitiva se não há uma gestão de qualidade preparada para suprir as necessidades das organizações.

O controle de qualidade na indústria "é um processo de delegação de responsabilidade e autoridade a atividade gerencial, porém mantendo meios para garantir resultados satisfatórios" (FEIGENBAUM, 1994).

Chaves, (1998, apud Corrêia, 2008), descreveu que "o objetivo do controle de qualidade é alcançar no produto um padrão de qualidade seja a curto ou em longo prazo sem perder sua essência original. Acompanhando e realizando medidas ou ações durante a produção, processamento, armazenamento e comercialização do produto”. 


\subsection{BPF - Boas práticas de fabricação}

Buscando uma boa vantagem comercial, empresarial no meio farmacêutico, as organizações visam melhorar seus processos produtivos e afins, de modo que tais processos recebam mais benefícios que os outros e melhorias que a empresa têm para se manter a frente de novos produtos, serviços e tecnologia no segmento.

As BPFs têm completa relação à sigla Good Manufacturing Practice (GMP), com a Food and Drug Administration (FDA). A sua origem teve surgimento no meio da década de 60 com o governo Americano, se deparando com grandes problemas em relação à higiene pessoal. Visto que o BPF visa à higiene-sanitária em setores produtivos e de processos nas organizações.

As Boas Práticas de Fabricação são práticas de higiene reconhecidas internacionalmente e recomendadas para o controle higiênico-sanitário, conhecidas desde a década de cinquenta do século passado, e que compreendem uma série de normas e procedimentos que devem ser atendidos para a produção. (NEVES; SANTOS; ROBBS, p. 10, 2009).

As BPFs são "um conjunto de normas obrigatórias que estabelecem e padronizam procedimentos e conceitos de boa qualidade para produtos, processos e serviços, visando atender aos padrões mínimos estabelecidos por órgãos reguladores governamentais nacionais e internacionais, cuja incumbência é zelar pelo bem-estar da comunidade" (PEREIRA FILHO; BARROCO, 2004).

Na tabela 1 são avaliados em tópicos os portes das empresas para a implantação do BPF.

Tabela 1: Coeficiente de correlação entre tópicos avaliados e porte das empresas respondentes. Porte da Empresa

\begin{tabular}{|c|c|c|c|}
\hline Motivo de Implantação & Pequena & Média & Grande \\
\hline Melhor relacionamento com os clientes & 0,49 & $\mathbf{0 , 0 3}$ & $-0,48$ \\
\hline Melhor qualidade do produto final & $\mathbf{0 , 4 1}$ & $-0,12$ & $-0,25$ \\
\hline $\begin{array}{l}\text { Melhor controle dos parâmetros dos processos e do } \\
\text { produto final }\end{array}$ & $\mathbf{0 , 2 6}$ & $-0,08$ & $-0,16$ \\
\hline $\begin{array}{l}\text { Melhor gestão da qualidade em termos } \\
\text { organizacionais }\end{array}$ & $\mathbf{0 , 2 6}$ & $-\mathbf{0 , 0 8}$ & $-0,16$ \\
\hline Redução de custos & 0,19 & $-0,17$ & $\mathbf{0 , 0 0}$ \\
\hline Obrigação da legislação vigente & $\mathbf{0 , 1 0}$ & $\mathbf{0 , 0 3}$ & $-0,12$ \\
\hline Melhor relacionamento com autoridades & $\mathbf{0 , 1 0}$ & $\mathbf{0 , 0 3}$ & $-0,12$ \\
\hline Melhoria da imagem da empresa & $\mathbf{0 , 0 0}$ & $-0,12$ & $\mathbf{0 , 1 3}$ \\
\hline Vantagem comercial da empresa & $\mathbf{0 , 0 0}$ & $-0,12$ & 0,13 \\
\hline
\end{tabular}


Fonte: CALARGE, SATOLO (2007).

Sobre as dificuldades encontradas em cada porte de empresa, destaca se:

A análise das dificuldades organizacionais permite verificar que as empresas de pequeno porte enfrentam maiores problemas, principalmente no que diz respeito ao desconhecimento dos funcionários sobre o sistema BPF, ao baixo envolvimento da alta administração e à insuficiência de instrução e treinamento da força de trabalho. (CALARGE; SATOLO, P. 388, 2007).

Algumas organizações passam por problemas para a implantação das boas práticas de fabricação, dificuldades como organizacionais, tecnológicas e financeiras citadas na tabela 2:

Tabela 2: Coeficientes de correlação entre aspectos que dificultam e o porte das empresas.

\begin{tabular}{llrcc} 
& & \multicolumn{3}{c}{ Porte das Empresas } \\
\cline { 2 - 5 } Dificuldade organizacional & & Pequena & Média & Grande \\
& Desconhecimento dos funcionários do sistema BPF & 0,33 & $-0,29$ & 0,00 \\
& Envolvimento de apenas parte da alta administração da empresa & 0,11 & $-0,10$ & 0,00 \\
& Instrução e treinamento insuficientes da força de trabalho & 0,11 & $-0,10$ & 0,00 \\
& Dificuldade no controle da documentação & 0,00 & 0,12 & $-0,13$ \\
& Dificuldade na elaboração de rotinas & 0,00 & 0,12 & $-0,13$ \\
& Dificuldade de entendimento da norma & $-0,26$ & 0,08 & 0,16 \\
& Dificuldade na elaboração da documentação exigida & $-0,33$ & 0,29 & 0,00 \\
\hline Dificuldade tecnológica & Instalações físicas da empresa apresentam-se inadequadas & 0,00 & $-0,24$ & 0,25 \\
& Equipamentos muito antigos & $-0,10$ & 0,31 & $-0,24$ \\
& Dificuldade de validação dos produtos & $-0,26$ & 0,08 & 0,16 \\
& Dificuldade de validação dos processos & $-0,33$ & 0,29 & 0,00 \\
& Falta de equipamentos de sofisticação tecnológica & $-0,33$ & 0,68 & $-0,41$ \\
& Dificuldade de validação dos métodos & $-0,41$ & 0,12 & 0,25 \\
& Dificuldades na aquisição de equipamentos laboratoriais & $-0,41$ & 0,48 & $-0,13$ \\
\hline Dificuldade financeira & Alto custo da consultoria & 0,26 & 0,08 & $-0,32$ \\
& Alto custo de implantação & 0,11 & 0,29 & $-0,41$ \\
& Priorização de investimentos em setores não ligados às BPFs & $-0,19$ & 0,17 & 0,00 \\
& Alto custo dos equipamentos & $-0,26$ & 0,53 & $-0,32$ \\
\hline
\end{tabular}

Fonte: CALARGE, SATOLO (2007).

\subsection{Processos existentes na produção de medicamentos veterinários}

A fabricação na indústria de medicamentos envolve a extração, purificação, síntese química e procedimentos de fermentação e o processamento farmacêutico para a preparação das drogas a granel em uma primeira etapa de processo, consequentemente o medicamento final surge como resultado de uma série de processos que dão formas e as dosagens de administração, envolvidos em embalagens convenientes ao uso prescrito. (SCHREVE, 1977).

De acordo com Schreve (1977) os medicamentos podem ser classificados para a apresentação de sua utilização, pelas reações e estruturas químicas (conversões químicas) 


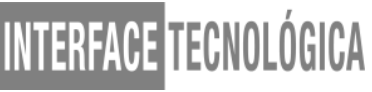

usadas nos processos de fabricações. Tais reações são usadas para obtenção de princípios ativos que são: alquilação, carboxilação, condensação, desidratação, ciclização, esterificação, halogenação, sulfonação e oxidação.

O fluxograma abaixo mostra a complexidade do estudo sobre a manufatura dos sistemas de aplicação de medicamentos veterinários e seus processos.

Ilustração 2: Fluxograma de extrusão continua a quente.

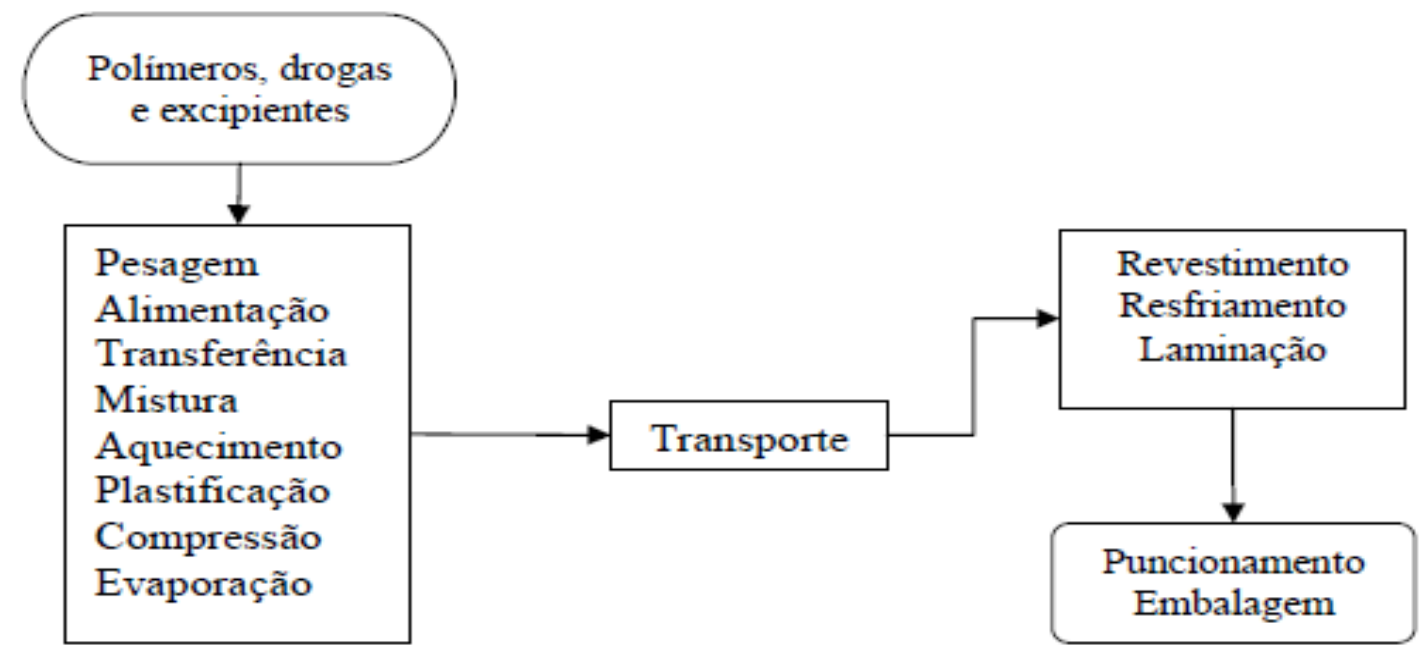

Fonte: Schreve (1977)

Tendo em vista as impossibilidades tecnológicas que as organizações possuem, estas têm um destaque de médios e grandes portes. Para empresas medianas o grande impasse é a falta de equipamentos adequados (sofisticação tecnológica), já as de grande porte a dificuldade central, é a infraestrutura fabril e as validações de métodos.

\subsection{Instalações e projetos}

Para ter qualidade na fabricação de medicamentos veterinários vários fatores têm interação entre si, tais como: instalações, condições ambientais e humanas e as normas que regem as organizações.

Sobre as instalações, a IN 4 (2007), alguns aspectos são apurados: construção do piso de trabalho em condições impermeáveis na parte interna o prédio, pisos de fácil lavagem e com polímeros antiderrapantes, internamente deve se ter vedação contra pragas (ratos, roedores e insetos). Os sistemas de esgoto e de água da empresa deve ter uma interligação de um sistema de reaproveitamento de resíduos de acordo com as normas vigentes. 


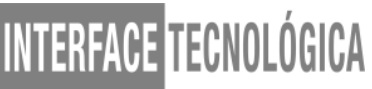

De acordo com Ribeiro (2009) os ambientes como: banheiros, vestiários e cozinhas (refeitórios) não podem ter comunicação direta com o local de processamento de medicamentos animais e alimentação animal, mantidos sempre limpos.

\subsection{Maquinários e utensílios}

Existem alguns materiais que não transmitem toxicidade, tais materiais como o aço, fibras e metais anticorrosivos são usados em máquinas e materiais que são usados no processo produtivo. Máquinas e materiais precisam ter muita resistência por serem usadas e lavadas a cada processo e a cada uso, e suas superfícies laváveis têm de ser planas e lisas para evitar sujidades e acúmulo de impurezas durante a limpeza.

\subsection{Produção}

$\mathrm{Na}$ etapa produtiva, os colaboradores e o pessoal precisam de capacitação e treinamento para desempenhar seu papel junto à indústria, e tendo supervisão a cada processo para não haver contaminação dos medicamentos veterinários. No local produtivo os medicamentos devem ficar longe de químicos, físicos e matérias microbiológicos.

\subsection{Matérias primas}

Para cada conjunto de matéria prima devidamente identificada com suas propriedades químicas, os fornecedores devem ter credenciais de qualidade higiênico-sanitária. Embalagens das matérias primas com sua embalagem original com seu rótulo diretamente do fabricante e sem problemas com avarias.

Recebimento de matéria prima é o início do controle de BPF, a mesma tende a ser identificada, codificada e ter um controle higiênico-sanitário no ato da armazenagem.

Como diz Manzalli (2006) todo o material que é enviado à empresa, embalagens e matérias primas deve ter seu armazenamento em paletes, estrados plásticos, sendo respeitado um espaço mínimo de armazenagem para garantir a ventilação e mobilidade.

\subsection{Produto acabado e a qualidade}




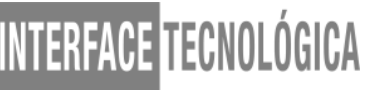

Todo o produto farmacêutico veterinário e outros produtos passam por testes laboratoriais do produto final.

O controle de qualidade da empresa deve atentar ao teste laboratorial atentando se em algumas informações básicas do produto e se o produto esta conforme igual ao teste previsto ao controle de qualidade. Teste de $\mathrm{pH}$, turbilidade, densidade e teste de pontos microbiológicos, para não ter sujidades, ferrugem e rótulos, e se o produto está devidamente estético ao consumidor final.

\subsection{Treinamentos de pessoal e visitante}

Antes de iniciar um processo, os colaboradores admitidos terão que passar por treinamentos higiênico-sanitários a fim de trabalharem nos seus devidos postos nas áreas produtivas. Os treinamentos têm de ser atualizados, periciados sempre que houver necessidade, alinhando com a motivação e resolvendo problemas diários na empresa.

Supervisores, auditores e pessoas interessadas para conhecer a empresa e seus processos são considerados visitantes. Os visitantes mesmo não sabendo as normas que regem a empresa, mesmo assim precisam ajustar os requisitos do BPF, usando jalecos, toucas e pro pé descartável em toda a área produtiva.

\subsection{Aferições de equipamentos de medição}

Os equipamentos que se situam nas áreas de processo e laboratórios precisam de calibrações e aferições periodicamente para evitar desvios padrão nas operações. Óleos e lubrificantes de máquina e equipamento não podem ser usados na manutenção usando o mesmo em excesso, prevenindo que caia resíduos nos medicamentos e afins.

\subsection{Documentos regulatórios}

De acordo com a Instrução Normativa $\mathrm{N}^{\circ} 4$ do MAPA (2007) em todo o processo produtivo as empresas devem documentar todos os registros e controles realizados no processamento, desde os inputs recém-chegados até os outputs finais. Documentos como reclamações, sugestões e elogios dos colaboradores devem ser disponíveis para consulta em ordem cronológica de arquivamento. 


\subsection{Controles de vetores e pragas}

Desde os tempos remotos da humanidade, as pragas já fazem seu papel de destruição, trazendo doenças, vírus e enfermidades aos seres humanos.

Problemas como restos de comida, água parada em áreas externas da empresa, acúmulo de poeiras e pó, matérias primas amontoadas em cantos, trazem a proliferação de insetos e roedores às embalagens trazendo essas pragas nos setores produtivos.

Medidas são necessárias para que essas pragas não entrem em setores produtivos, tais como aplicando se telas milimetradas, portas com tela para não entrada de mosquitos, portas com sistema de fechamento automático rápido e proteções de borrachas em portas e batentes, colocação de ralos onde não se tem um sistema sifão. Lâmpadas compostas de sódio evitam que insetos se proliferem ao redor delas no período noturno.

Não tendo eficácia em controlar e aniquilar essas pragas na empresa, à mesma deve consultar e contratar empresas especializadas a fim de amenizar e controlar essas pragas, utilizando pesticidas e produtos químicos para o controle.

\subsection{Abastecimento e tratamento de água e afluentes}

A empresa deverá ter um abastecimento de água potável em sua instalação, ter o controle de temperatura e de contaminação, ter uma caixa de água apropriada industrialmente para armazenagem de água potável para consumo humano e fontes de filtragem para processos produtivos, encanamentos em perfeito estado de conservação para evitar contaminação antes de qualquer uso.

A qualidade da água na empresa tem de ser: limpa, inodoro, livre de microrganismos e não corrosiva. (GONÇALVES, 2002)

\section{PROPOSTA DE MELHORIA COM O USO DAS BOAS PRÁticAs DE FABRICAÇÃO}

O controle de boas práticas de fabricação deve ser mantido e melhorado nas indústrias de produção de medicamentos veterinários visto que essa metodologia é aplicada no processo 
produtivo em baixa, media e larga escala, respeitando os padrões estabelecidos por cada empresa, independentemente de seu porte.

Para que os dados e controle que a qualidade mantém no processo, essas informações devem ser mantidas e supervisionadas como de costume para não haver não conformidades, feitas com a emissão de ordens de produção, check lists e documentos que seguem o BPF.

Alguns problemas tais como: falta de comprometimento de pessoal, desafios na implantação, infraestrutura escassa e custos são encontrados nas empresas desse ramo, e por isso as boas práticas de fabricação e a função da qualidade servem de alicerce a essas empresas.

Na ilustração 3 são mostradas as melhorias com o uso das boas práticas de fabricação.

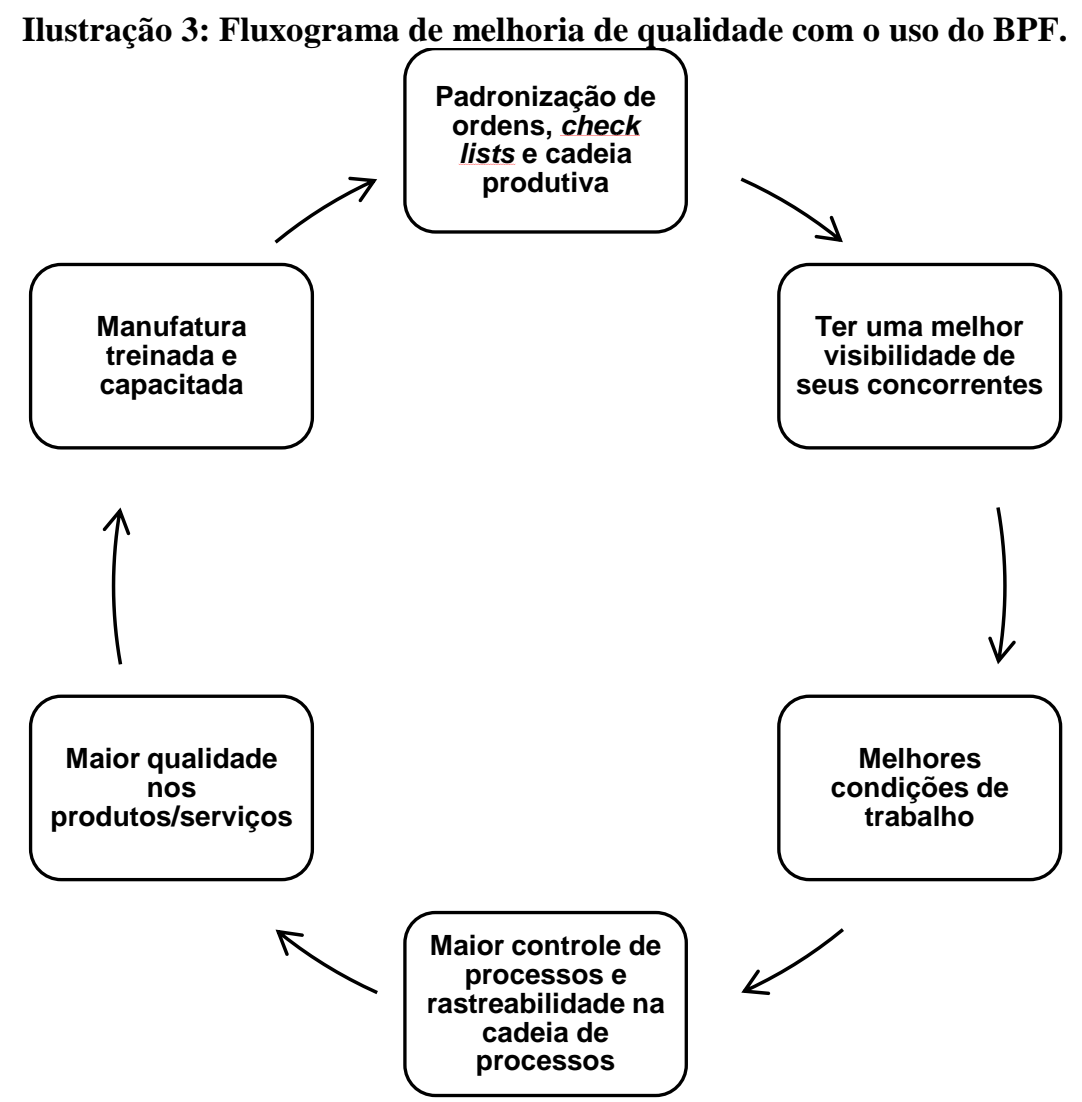

Fonte: Elaborado pelo autor (2017).

Outra função são os check lists (documentos de toda a cadeia produtiva em relação á qualidade) que mostra e monitora todos os processos dentro da manufatura, e documentos regulatórios são essenciais e mostram com clareza a empresa de acordo com os órgãos que regem a sua funcionalidade. E por meios de audições as empresas são auditadas periodicamente para manter o controle das certificações de qualidade. 


\section{RESULTADOS E DISCUSSÃO}

O compromisso com a qualidade é uma das principais preocupações das organizações no processo produtivo, sendo redobrada em indústrias farmacêuticas a fim de garantir a satisfação dos clientes. Para se garantir uma empresa com qualidade em seus processos, existem metodologias que cumprem a qualidade, no caso as boas práticas de fabricação para uma produção eficiente e segura.

O presente estudo mostrou a presença das boas praticas de fabricação e sua essencialidade nas organizações, sendo uma metodologia satisfatória nesse ramo de indústria, visto que para ter competitividade e colocar seu preço no mercado, essas empresas necessitam de qualidade nos seus processos e com uma metodologia do BPF fica mais fácil obter seus objetivos com clareza e responsabilidade.

\section{CONSIDERAÇÕES FINAIS}

Empresas de todo o mundo utilizam de métodos, metodologias e estudos para obter competitividade no mercado, buscam a qualidade para obter certificações e com o BPF essa amplitude, aumenta permitindo uma análise estruturada da função-produção de acordo com os parâmetros que as boas práticas de fabricação permitem a essas organizações.

Usando a qualidade em seu favor, o Brasil se encontra entre os cinco maiores do mundo, com o maior mercado bovino comercial, as perspectivas de crescimento em medicamentos veterinários só crescem, fazendo os empresários a buscarem novas técnicas e novos interesses junto com a qualidade, com isso, as boas práticas de fabricação são o inicio de uma jornada de qualidade nas organizações, aumentando sua competitividade no cenário mundial de medicamentos para animais.

\section{REFERÊNCIAS}

BRASIL. Ministério da Agricultura, Pecuária e Abastecimento. Instrução Normativa $\mathbf{N}^{\mathbf{0}} \mathbf{4}$, 23 fev. 2007. Diário Oficial da União, Brasília, 23 fev. 2007.

CAPANEMA, Luciana Xavier de Lemos et al. Panorama da indústria farmacêutica veterinária. BNDES Setorial, n. 25, p. 157-173, mar. 2007. 


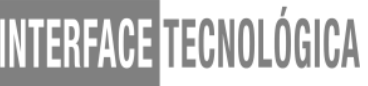

CHAVES, L.S. Avaliação da implementação de uma ferramenta de controle-BPF em empresas registradas junto CISPOA. 2014. Trabalho de Conclusão de curso 45 (Especialização em Produção, Tecnologia e Higiene em Produtos de Origem Animal) Universidade Rio Grande do Sul. Porto Alegre 2014.

CORRÊA, J. G. F., A Importância da Higiene de Manipuladores para a Qualidade dos Alimentos. Monografia (Especialização em Higiene e Inspeção de Produtos de Origem Animal) - Pró- Reitoria de Pesquisa e Pós-Graduação - Universidade Castelo Branco - UNB. Campo Grande. 2008.

FEIGENBAUM, A. V. Controle da qualidade total, Volume I. São Paulo: Makron Books, 1994.

FOOD AND DRUG ADMINISTRATION. History of the FDA. Disponível em:

〈http://www.fda.gov/oc/history/historyoffda/default.htm>. Acesso em: 10 ago. 2017.

PEREIRA FILHO, W.R.; BARROCO, R. (2004) - Gestão da qualidade na indústria farmacêutica. In: OLIVEIRA, O.J. (org.) Gestão da qualidade: tópicos avançados. São Paulo: Thomson, 2004. cap.15. p.211-215.

PINTO, M.S. Garantia da qualidade na indústria cosmética. São Paulo: Cengage Lenning, 2012.

SHREVE, R.N.; BRINK Jr, J.A. Indústrias de Processos Químicos. 4.ed. Rio de Janeiro: Guanabara Koogan, 1980.

WAACK, Roberto Silva. Fusões e aquisições na indústria farmacêutico-veterinária. Revista de Gestão da Universidade de São Paulo, São Paulo, v. 7, n. 3, p. 81-98, 2000. 